\title{
Perioperative care following strabismus surgery for the patient with severe congenital neutropenia
}

\author{
Joon Ho Park, Kyoung Min Kang, Bo Young Chun* \\ Department of Ophthalmology, School of Medicine, Kyungpook National University, Daegu, South Korea; \\ *Corresponding Author: byjun424@hotmail.com
}

Received 24 January 2013; revised 28 February 2013; accepted 10 March 2013

\begin{abstract}
A 10-year-old girl presented with esotropia. She had 35 prism diopters of esodeviation at far and near with left inferior oblique muscle overaction (+3). Cycloplegic refraction was +0.5D OD and +0.75D OS, and visual acuity was 20/25 in each eye. The patient was being treated with G-CSF (Leufokine $^{\circledR}$ ) due to her severe congenital neutropenia (WBC 3350, neutrophil 7.3\%, and ANC 170/ul). Despite of low ANCs, we decided to perform a surgery because the patient and her parents strongly wanted it. Prophylactic topical antibiotics (Cravit ${ }^{\circledR}$, levofloxacin $0.5 \%$ ) were applied four times a day for three days prior to the surgery. We sterilized the periocular skin with povidone-iodine $10 \%$ and placed povidone-iodine $5 \%$ drops in the cul-de-sac preoperatively. Strabismus surgery was performed. After surgery, profuse irrigation with the entire bottle of topical antibiotics (Vigamox ${ }^{\circledR}$, moxifloxacin hydrochloride $0.5 \%$ ) was performed for each eye. We prescribed topical and highly potent systemic antibiotics for 1 week. This is the first case report to describe the successful perioperative antisepsis care following strabismus surgery for a patient with severe congenital neutropenia. Prevention of postoperative infection in granulocytopenic patients could be achieved using antibiotic agents which provide the greatest spectrum of coverage against both Gram-positive and Gram-negative infections.
\end{abstract}

Keywords: Congenital Neutropenia; Perioperative Care; Strabismus Surgery

\section{INTRODUCTION}

Periocular infections after strabismus surgery are rare but potentially serious. These infections include cellulites, subconjunctival and sub-Tenon's abscesses, and endo- phthalmitis $[1,2]$. The incidence of periocular infection is assumed to be one case per 1100 surgeries [3]. All infections are potentially vision threatening and are more prone to occur in patients with immune deficiency. Severe congenital neutropenia is characterized by absolute neutrophil counts (ANCs) consistently below 200/ul, with recurrent, severe infections developing in the first months of life [4]. In this report, we describe a case of the successful perioperative care following strabismus surgery for a patient with severe congenital neutropenia.

\section{CASE REPORT}

A 10-year-old girl presented with esotropia, which had been present since her first year of life, in September 2009. Upon examination, she had 35 prism diopters (PD) of esodeviation at far and near with left inferior oblique muscle overaction (IOOA) (+3) (Figure 1). Cycloplegic refraction was $+0.5 \mathrm{D}$ OD and +0.75D OS, and visual acuity was 20/25 in each eye. The results of optic disc and retinal examinations were normal in both eyes.

The patient was being treated with G-CSF (Leufokine ${ }^{\circledR}$ ) due to her severe congenital neutropenia (WBC 3350, neutrophil 7.3\%; normal: 40\% - 74\%, and ANC 170/ul). The patient had a history of recurrent gingivitis and cellulitis and was admitted to the pediatric department several times due to low ANCs. The authors suggested delaying her surgery due to the high risk of postoperative infection. However, the patient and her parents strongly insisted on the surgery, due to the emotional stress experienced by the patient on account of her esodeviated eyes. Eventually, authors determined to perform the surgery not only the family and the patient strongly insisted but also her pediatrician allowed her the surgery on con-

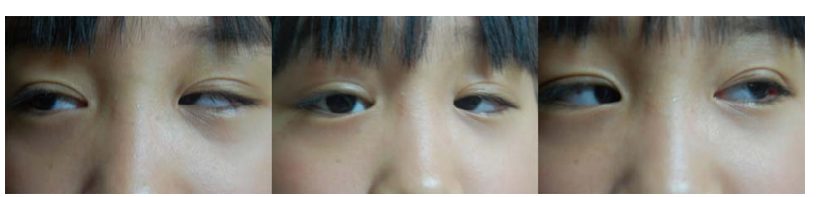

Figure 1. Preoperative photos demonstrating esotropia of 35 prism diopters. 
dition of close observation due to the fact that her ANC level was higher than ever.

Prior to surgery, prophylactic topical antibiotics (Cravit ${ }^{\circledR}$, levofloxacin $0.5 \%$ ) were applied four times a day for three days prior to the surgery. The surgery was performed under general anesthesia by the author (BYC).

We sterilized the periocular skin with povidone-iodine $10 \%$ and placed povidone-iodine $5 \%$ drops in the cul-desac preoperatively. After limbal incision, bilateral medial rectus muscle recession $(4.5 \mathrm{~mm})$ was performed. Then, through infero-temporal conjunctival incision, the left inferior oblique muscle was anteriorized to $1 \mathrm{~mm}$ posterior to the inferior rectus muscle insertion. After conjunctival closure with vicryl 6-0, profuse irrigation with the entire bottle of topical antibiotics (Vigamox ${ }^{\circledR}$, moxifloxacin hydrochloride $0.5 \%$ ) was performed for each eye.

On the day of the surgery, systemic antibiotics (Maxifime $^{\circledR}$, 4th generation of cephalosphorin) were administered intravenously. The patient was monitored closely to detect any changes upon clinical examination, including best-corrected visual acuity, pupillary reaction, slit lamp and dilated fundus examinations, which were performed every 8 hours after the surgery until discharge.

On the first postoperative day, there was no evidence of local postoperative infection. In addition, there was no interval change in the general condition or vital signs between prior to and after the surgery. The patient was discharged on the second postoperative day. The patient was prescribed oral antibiotics (Banan ${ }^{\circledR}$, quinolone) and topical antibiotics (Vigamox ${ }^{\circledR}$, every 4 hours) for 1 week and advised to visit the clinic everyday until the seventh postoperative day. On the postoperative 2 weeks, the patient was orthophoric and did not show any systemic or local signs of infection (Figure 2). On the last follow-up, 6 months after the surgery, the patient remained without symptom or sign suggestive of a postoperative infection.

\section{DISCUSSION}

Periocular infections after strabismus surgery are rare but potentially serious. These infections include cellulitis, subconjunctival and sub-Tenon's abscesses, myositis and endophthalmitis [2,3]. Ing reported that cellulitis occurred in 1 per 1900 cases and that endophthalmitis occurred in 1 per 30,000 cases [4].

Despite the low incidence of periocular infection after strabismus surgery, the condition is potentially vision threatening and is more prone to occur in patients with

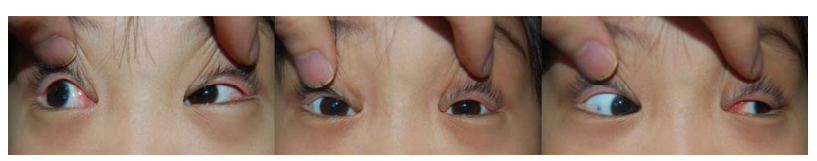

Figure 2. Photos demonstrate orthophoria and no sign of postoperative infection at 2 weeks postoperatively. immune deficiency. Children with immune deficiency may also become strabismic, and they will need additional perioperative care to prevent infection related to surgery.

In this report, we describe a case of the successful perioperative care following strabismus surgery for the patient with severe congenital neutropenia. Severe congenital neutropenia is characterized by absolute neutrophil counts (ANCs) consistently below 200/ul, with recurrent, severe infections developing in the first months of life [1].

To reduce the amount of normal flora in the eyes, the authors prescribed a combination of preoperative antisepsis, including a 3-day preoperative application of topical antibiotics and instillation of 5\% povidone-iodine solution into the fornix prior to surgery. Povidone-iodine has been found to decrease the number of bacterial colonies by $91 \%$ when just one to two drops are administered as a preoperative surgical preparation [5,6]. The use of povidone-iodine reduces the incidence of postoperative infections in some intraocular surgeries by decreasing the bacterial load present in the conjunctival fornices $[2,7,8]$.

To reduce the risk of postoperative infection, the authors irrigated the eyes with topical antibiotics after surgery, and prescribed topical and highly potent systemic antibiotics for 1 week. Moxifloxacin $0.5 \%$ (Vigamox $^{\circledR}$ ) is the most broad-spectrum agents commercially available to treat or prophylax ophthalmic infections [9]. It covers bacterial resistance to the second- and third-generation fluoroquinolones, is more potent for gram-positive bacteria, and is equally potent for gram-negative bacteria [10]. Considering that Gram positive cocci are the predominant microorganisms causing infections in granulocytopenic patients, we prescribed the fourth-generation cephalosporin (Maxifeme ${ }^{\circledR}$ ) postoperatively [11]. Compared with the third-generation cephalosporins, the fourthgeneration cephalosporins are more active and possess enhanced activity against Gram-positive bacteria and Enterobacteriaceae [11].

This is the first case report to describe the successful perioperative antisepsis care following strabismus surgery for a patient with severe congenital neutropenia. Prevention of postoperative infection in granulocytopenic patients could be achieved using antibiotic agents which provide the greatest spectrum of coverage against both Gram-positive and Gram-negative infections.

\section{REFERENCES}

[1] Boxer, L.A. and Newburger, P.E. (2007) A molecular classification of congenital neutropenia syndromes. Pediatr Blood Cancer, 49, 609-614. doi:10.1002/pbc.21282

[2] Brenner, C., Ashwin, M., Smith, D. and Blaser, S. (2009) Sub-Tenon's space abscess after strabismus surgery. 
Journal of AAPOS, 13, 198-199. doi:10.1016/j.jaapos.2008.10.010

[3] Basheikh, A. and Superstein, R. (2009) A child with bilateral orbital cellulitis one day after strabismus surgery. Journal of AAPOS, 13, 488-490. dx.doi:10.1016/j.jaapos.2009.05.016

[4] Ing, M.R. (1991) Infection following strabismus surgery. Journal of Ophthalmic Nursing \& Technology, 10, 211214.

[5] Koederitz, N.M., Neely, D.E., Plager, D.A., Boehmer, B., Ofner, S., Sprunger, D.T., et al. (2008) Postoperative povidone-iodine prophylaxis in strabismus surgery. Journal of AAPOS, 12, 396-400. doi:10.1016/j.jaapos.2007.10.005

[6] Apt, L., Isenberg, S., Yoshimori, R. and Paez, J.H. (1984) Chemical preparation of the eye in ophthalmic surgery: III. Effect of povidone-iodine on the conjunctiva. Archives of Ophthalmology, 102, 728-729. doi:10.1001/archopht.1984.01040030584025

[7] Palamar, M., Uretmen, O. and Kose, S. (2005) Orbital cellulitis after strabismus surgery. Journal of AAPOS, 9,
602-603. doi:10.1016/j.jaapos.2005.05.009

[8] Recchia, F.M., Baumal, C.R., Sivalingam, A., Kleiner, R., Duker, J.S. and Vrabec, T.R. (2000) Endophthalmitis after pediatric strabismus surgery. Archives of Ophthalmology, 118, 939-944.

[9] Jensen, M.K., Fiscella, R.G., Moshirfar, M. and Mooney, B. (2008) Third- and fourth-generation fluoroquinolones: Retrospective comparison of endophthalmitis after cataract surgery performed over 10 years. Journal of Cataract \& Refractive Surgery, 34, 1460-1467. doi:10.1016/j.jcrs.2008.05.045

[10] Mather, R., Karenchak, L.M., Romanowski, E.G. and Kowalski, R.P. (2002) Fourth generation fluoroquinolones: New weapons in the arsenal of ophthalmic antibiotics. American Journal of Ophthalmology, 133, 263-266.

[11] Garau, J., Wilson, W., Wood, M. and Carlet, J. (1997) Fourth-generation cephalosporins: A review of in vitro activity, pharmacokinetics, pharmacodynamics and clinical utility. Clinical Microbiology and Infection, S1, S87S101. doi:10.1111/j.1469-0691.1997.tb00649.x 